\title{
Raiva humana transmitida por cães: áreas de risco em Minas Gerais, Brasil, 1991-1999
}

\author{
Human rabies transmitted by dogs: \\ risk areas in Minas Gerais, Brazil, 1991-1999
}

Cristiana Ferreira Jardim de Miranda 1

José Ailton da Silva 2

Élvio Carlos Moreira 2

\footnotetext{
1 Diretoria Metropolitana de Saúde, Secretaria de Estado de Saúde de Minas Gerais. Rua Guajajaras 1956, Belo Horizonte, $M G$ 30180-101, Brasil. kikijardim@uol.com.br 2 Departamento de Medicina Veterinária Preventiva, Escola de Veterinária, Universidade Federal de Minas Gerais. Av. Antônio Carlos 6627, Belo Horizonte, $M G$ 30123-970, Brasil. jjasilva@vet.ufmg.br elviocm@vet.ufmg.br
}

\begin{abstract}
A retrospective study based on observation with the objective of identifying and characterizing the different risk areas for rabies transmission by dogs took place in Minas Gerais State, Brazil, from 1991 to 1999. Indicators confirmed occurrences of canine and feline rabies, notification of human rabies, and administration of appropriate vaccination. The Minas Gerais State Health System is divided into 25 Regional Health Centers, which are linked to the State Health Department (SES-MG). These Health Centers were utilized in the study. The results of 2,845 records of laboratory diagnosis for canine, feline, and human rabies were analyzed. Consolidated SES-MG reports from 1997 to 1999 for rabies vaccination and notification records for cases of human rabies from the National Health Foundation (FUNASA) were also used. In order to verify the local reality, a semi-structured interview with each regional program director was conducted. Minas Gerais presents four different risk modalities, classified as zero, low, medium, and high.
\end{abstract}

Key words Rabies; Dogs; Risk Zone; Retrospective Studies; Zoonosis

Resumo Com o objetivo de identificar e caracterizar as diferentes áreas de risco para a raiva humana transmitida por cães em Minas Gerais, realizou-se um estudo observacional retrospectivo quali-quantitativo, no período de 1991 a 1999. Utilizou-se a divisão do estado em 25 Diretorias Regionais de Saúde (DRS), vinculadas à Secretaria de Estado da Saúde de Minas Gerais (SES-MG). Os indicadores estudados foram a estimativa de população canina, a ocorrência de raiva canina e felina confirmada por exames laboratoriais, raiva humana notificada, coberturas vacinais e a infra-estrutura do serviço na DRS. Foram analisados os resultados de $2.845 \mathrm{fi}$ chas de diagnóstico laboratorial para raiva canina, felina e humana, provenientes dos laboratórios de referência para a raiva, relatórios de consolidados de vacinação anti-rábica animal da SES-MG dos anos de 1997 a 1999 e fichas de notificação dos casos de raiva humana da Fundação Nacional de Saúde (FUNASA). Realizou-se entrevista semi-estruturada com cada responsável regional pelo programa de controle da raiva. Os resultados permitem classificar Minas Gerais em quatro diferentes modalidades de risco: nulo, baixo, médio e alto.

Palavras-chave Raiva; Cães; Zona de Risco; Estudos Retrospectivos; Zoonoses 


\section{Introdução}

De todas as zoonoses, a raiva é a mais temida delas, sendo o cão ainda o principal transmissor para o homem. O Programa de Profilaxia da Raiva, criado em 1973 pelo Ministério da Saúde (MS), prevê como principal medida de controle da doença, a vacinação em massa de cães e gatos com o objetivo de se deter o ciclo de transmissão do vírus. Concomitante à vacinação animal, observa-se a descentralização do tratamento humano. O controle da circulação do vírus é realizado através da captura de cães errantes e observação de animais agressores, e monitorado através de exames laboratoriais específicos para a detecção do vírus rábico. As medidas de controle iniciam-se nas regiões metropolitanas e paulatinamente vão atingindo o interior. Dessa forma, na década de 90, a vacinação de cães e gatos já atingiu toda a área do espaço mineiro, mas ainda há persistência na circulação do vírus em algumas áreas e perdas humanas por essa doença.

Os fatores sociais funcionam como facilitadores ou empecilhos para a dispersão do vírus em uma determinada área. Quanto menor a situação de desenvolvimento local, maior é a promiscuidade observada na relação homem/animal e menores também os cuidados sanitários tomados.

Em vista das várias realidades observadas em Minas Gerais, este estudo pretende identificar e caracterizar as diferentes áreas de risco para a transmissão da raiva humana por cães, em virtude deste ainda ser o principal transmissor da doença ao homem, procurando nortear possíveis mudanças de conduta nas ações de controle e erradicação da doença.

\section{Material e métodos}

A área de estudo para este trabalho foi o Estado de Minas Gerais, que possui hoje 853 municípios. Corresponde a $6,89 \%$ do território nacional, com superfície de $588.383,6 \mathrm{~km}^{2}$. A população residente segundo o Censo 2000 é de 17.835.488 habitantes, sendo 14.623 .990 residentes em zona urbana e 3.211.498 na zona rural (IBGE, 2001).

Os serviços de saúde no estado são descentralizados em 25 Diretorias Regionais de Saúde (DRS), ligadas à Secretaria de Estado da Saúde (SES-MG) e que atuam diretamente junto às Secretarias Municipais de Saúde.

Os dados da raiva foram obtidos a partir dos relatórios enviados pelos laboratórios de referência para diagnóstico de raiva em Minas
Gerais. Foram utilizados todos os registros de diagnóstico para as espécies canina e humana, durante o período compreendido entre janeiro de 1991 e dezembro de 1999. Realizou-se uma entrevista semi-estruturada (Triviños, 1987) com os responsáveis regionais do Programa de Profilaxia da Raiva em cada DRS, por um único entrevistador. Os pontos básicos da entrevista foram a ocorrência de raiva humana, canina e felina, a vacinação em massa, o controle de cães errantes, a observação de animais agressores e o atendimento anti-rábico humano. $\mathrm{O}$ cálculo de estimativa utilizado nos planos oficiais de controle da Raiva até 1999 foi o de $10 \%$ da população humana residente. Neste trabalho, foi utilizada uma estimativa extra-oficial calculada em $17 \%$ da população humana, ou um cão para cada seis habitantes (OPAS, 1992), exceto na Diretoria Metropolitana de Saúde (DMS), onde a população foi anteriormente estimada em um cão para cada oito habitantes por Silva et al. (1982), e confirmada por censo canino realizado pela Prefeitura Municipal de Belo Horizonte no ano de 2000 (Secretaria Municipal de Saúde de Belo Horizonte, 2000). A população de Belo Horizonte corresponde a $51 \%$ do total da Regional, o que faz com que a população canina estimada para a DMS seja de um cão para cada sete habitantes ou $14 \%$. O período de análise das coberturas vacinais foi o de 1997 a 1999, obtidas junto à Coordenadoria de Zoonoses da SES-MG (2000).

De acordo com as informações obtidas, as DRS foram classificadas para a raiva humana com transmissão por cães e gatos, como "risco nulo ou ausente", "baixo risco", “médio risco”, e "alto risco", conforme as recomendações da Organização Pan-Americana da Saúde (PAHO, 1999).

- Risco nulo ou ausente: ausência de casos caninos e/ou felinos nos últimos três anos, cobertura vacinal $\geq 75 \%$, controle de cães errantes nos maiores centros urbanos, ausência de raiva canina e/ou felina nas áreas limítrofes, observação de animais agressores e envio de amostras de animais suspeitos, atendimento anti-rábico humano e presença de fatores ambientais naturais ou artificiais que dificultem a propagação do vírus.

- Baixo risco: ausência de raiva canina ou felina autóctone nos últimos três anos, ou raiva canina e/ou felina diagnosticada com realização de medidas de controle (bloqueio e controle dos contatos humanos e animais) e não ocorrência de casos secundários; cobertura vacinal $\geq 75 \%$, controle de cães vadios, observação de animais agressores, presença de raiva canina e/ou felina nas áreas vizinhas, observação de 
animais agressores e envio de amostras de animais suspeitos, atendimento anti-rábico humano e presença de fatores ambientais naturais ou artificiais que dificultem a propagação do vírus.

- Médio risco: ausência de raiva canina ou felina nos últimos três anos, cobertura vacinal $\leq 75 \%$, ausência de amostras canina e/ou felina nos últimos três anos, ausência de controle de cães vadios com observação de animais agressores, presença de raiva canina e/ou felina nas áreas vizinhas, presença de raiva canina e/ou felina com realização de medidas de controle (bloqueio de foco e controle dos contatos humanos e animais), atendimento anti-rábico humano e presença fatores ambientais que facilitem a propagação e/ou manutenção do vírus.

- Alto risco: presença de raiva canina e/ou felina autóctone e persistente por mais de um ano com confirmação laboratorial, cobertura vacinal $\leq$ de $75 \%$, ausência de outras medidas de controle, atendimento anti-rábico humano ineficaz e presença fatores ambientais que facilitem a propagação e/ou manutenção do vírus.

Os dados foram agrupadas utilizando-se o editor de texto Word versão 97, o programa Epi Info versão $6.04 \mathrm{~b}$, as tabelas e gráficos na planilha eletrônica Excel versão 97 e os mapas no programa Tabwin versão 1.4, sendo plotado nas bases Minas Gerais 1991, 1994 e 1997, obtidas no Departamento de Informática do SUS (DATASUS, 2000).

\section{Resultados}

A raiva canina ainda é endêmica em algumas áreas do estado e em muitos casos o diagnóstico é apenas clínico, devido à dificuldade de enviar amostras para os laboratórios de referência que se localizam na capital. Nas áreas onde persiste a raiva canina, a identificação do "cão zangado" por parte da comunidade é comum, o que contribui para a diminuição dos casos humanos - a população está ciente da necessidade do tratamento.

Dos 2.465 registros de amostras caninas enviadas aos laboratórios de referência para raiva em Minas Gerais, $47,42 \%$ do total de amostras enviadas são oriundos da DMS, seguidos pela DRS Uberlândia com 7,95\% dos registros. A média do interior é de seis amostras anuais por DRS, distribuídas de forma não uniforme (Tabela 1 ).

$\mathrm{O}$ vírus permanece circulando durante todo o período nas DRS de Diamantina, Governador Valadares, Pedra Azul e Teófilo Otoni. A partir de 1998, a DRS Manhumirim passa de

\begin{tabular}{|c|c|c|c|c|}
\hline \multicolumn{5}{|c|}{$\begin{array}{l}\text { Distribuição de amostras caninas submetidas a exames para raiva, por Diretoria } \\
\text { Regional de Saúde. Minas Gerais, Brasil, 1991-1999. }\end{array}$} \\
\hline $\begin{array}{l}\text { Diretoria Regional } \\
\text { de Saúde }\end{array}$ & $\begin{array}{l}\text { Amostras } \\
\text { positivas }\end{array}$ & $\begin{array}{l}\text { Amostras } \\
\text { totais }\end{array}$ & $\begin{array}{l}\text { Positivos } \\
\text { (\%) }\end{array}$ & $\begin{array}{l}\text { Total de } \\
\text { amostras (\%) }\end{array}$ \\
\hline Alfenas & 1 & 34 & 2,94 & 1,38 \\
\hline Barbacena & 0 & 25 & 0,00 & 1,01 \\
\hline Coronel Fabriciano & 1 & 72 & 1,39 & 2,92 \\
\hline Diamantina & 15 & 33 & 45,45 & 1,34 \\
\hline Divinópolis & 0 & 139 & 0,00 & 5,64 \\
\hline Governador Valadares & 50 & 89 & 56,18 & 3,61 \\
\hline Itabira & 2 & 96 & 2,08 & 3,89 \\
\hline Ituiutaba & 0 & 3 & 0,00 & 0,12 \\
\hline Juiz de Fora & 2 & 79 & 2,53 & 3,20 \\
\hline Leopoldina & 0 & 12 & 0,00 & 0,49 \\
\hline Manhumirim & 4 & 24 & 16,67 & 0,97 \\
\hline Metropolitano & 0 & 1.169 & 0,00 & 47,42 \\
\hline Montes Claros & 12 & 81 & 14,81 & 3,29 \\
\hline Passos & 0 & 25 & 0,00 & 1,01 \\
\hline Patos de Minas & 1 & 135 & 0,74 & 5,48 \\
\hline Pedra Azul & 16 & 20 & 80,00 & 0,81 \\
\hline Ponte Nova & 0 & 20 & 0,00 & 0,81 \\
\hline Pouso Alegre & 0 & 30 & 0,00 & 1,22 \\
\hline São João Del Rei & 0 & 8 & 0,00 & 0,32 \\
\hline Sete Lagoas & 1 & 45 & 2,22 & 1,83 \\
\hline Teófilo Otoni & 50 & 88 & 56,82 & 3,57 \\
\hline Ubá & 0 & 10 & 0,00 & 0,41 \\
\hline Uberaba & 0 & 3 & 0,00 & 0,12 \\
\hline Uberlândia & 0 & 196 & 0,00 & 7,95 \\
\hline Varginha & 0 & 29 & 0,00 & 1,18 \\
\hline Total & 155 & 2.465 & 6,29 & 100,00 \\
\hline
\end{tabular}

área silenciosa a positiva. A DRS Montes Claros apresentou positividade persistente no período de 1991-1994. As DRS de Itabira, Juiz de Fora, Patos de Minas e Sete Lagoas apresentaram casos isolados, controlados por bloqueio.

Na Figura 1, observa-se a distribuição da raiva canina durante o período de estudo.

Há uma tendência no aumento do envio de amostras para diagnóstico laboratorial, principalmente no último triênio do período de estudo (Figura 2), o que levou a um aumento nas taxas de incidência de raiva canina no mesmo período (Tabela 2).

A captura de cães errantes é uma prática pouco utilizada. Na maioria dos casos, observa-se a retirada de animais doentes por solicitação do proprietário, principalmente em áreas endêmicas para leishmaniose visceral.

Nos 33 casos de raiva humana notificados durante o período (Tabela 3), 21 foram transmitidos por cães $(63,64 \%)$. Desses casos, houve 
Figura 1

Distribuição de amostras caninas positivas para raiva. Minas Gerais,

Brasil, 1991-1999.
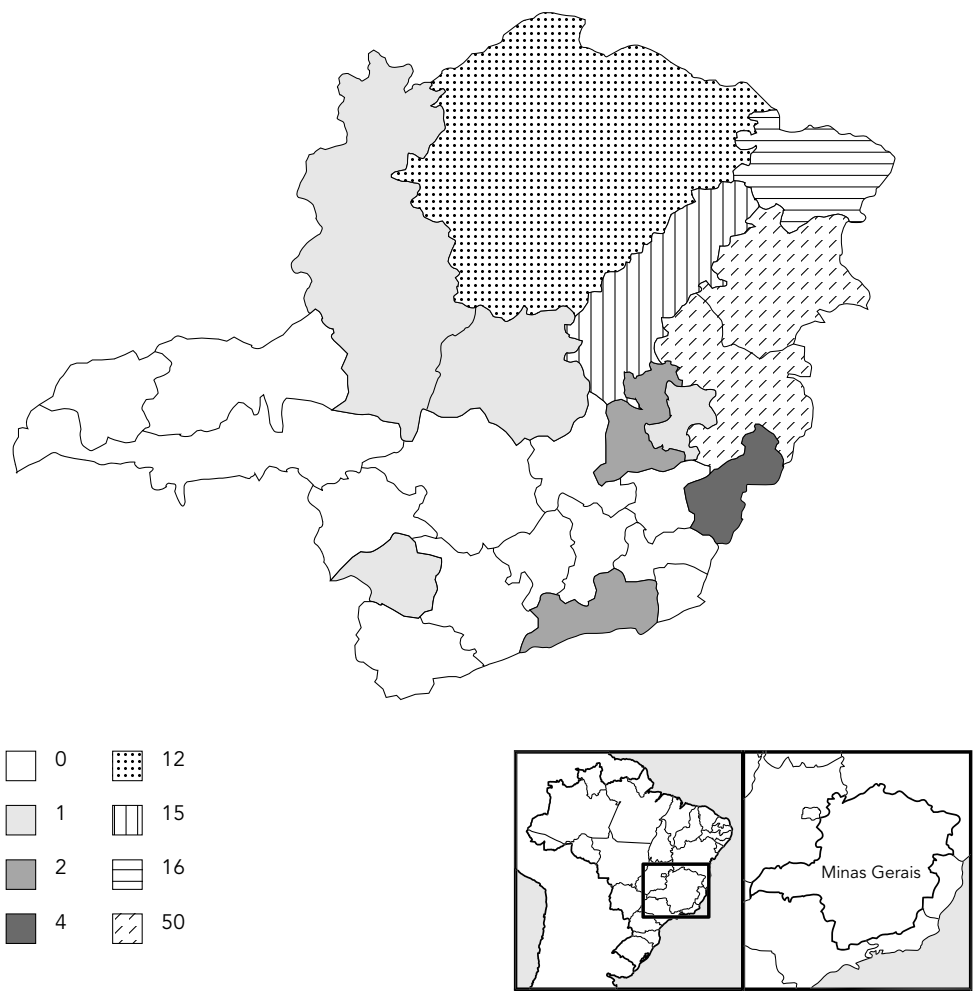

uma procura pelo atendimento médico pós-exposição no sistema de saúde privado, onde não houve indicação de tratamento, apesar de estar em área endêmica. Em apenas um dos casos (notificado pela DRS São João Del Rei), a investigação feita durante a pesquisa não conseguiu estabelecer uma correlação clínico-epidemiológica para raiva, apesar de haver exame laboratorial intra-vitam positivo. Nesse caso não houve confirmação laboratorial post-mortem.

O morcego vem como segundo maior transmissor dos casos humanos $(27,27 \%)$. O controle de morcegos hematófagos está sob responsabilidade do Ministério da Agricultura e Secretarias Estaduais de Agricultura. Não há um programa para controle das demais espécies e nem um específico do MS para controle dos mesmos.

Observa-se na Figura 3 que, com exceção do caso da DRS São João Del Rei, a distribuição da raiva humana transmitida por cães coincide com as áreas de maior incidência da raiva canina.

O atendimento anti-rábico humano está disponível em quase todos os municípios do Estado, e naqueles municípios em que não há atendimento médico específico, a vacina é enviada para tratamento dos pacientes em Posto de Saúde local após atendimento em município mais próximo que seja referência para o Programa na região. A aplicação do soro é feita em cidades-pólo das DRS, por sua necessidade de ambiente hospitalar. A prescrição da vacina

Figura 2

Freqüência no envio de amostras para diagnóstico laboratorial de raiva. Minas Gerais, Brasil, 1991-1999.

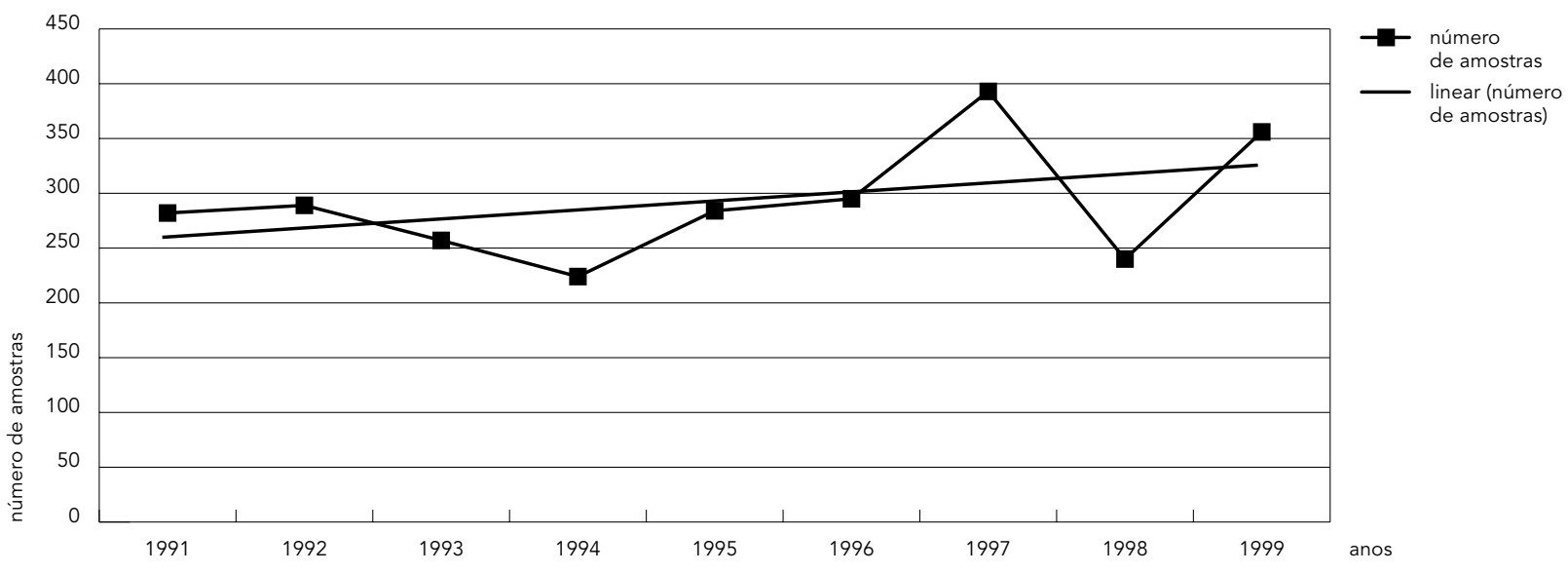


é estrita à classe médica. Os treinamentos para as equipes de atendimento não estão sendo feitos de modo rotineiro e são exclusivos aos serviços públicos.

A principal medida de impacto para o controle da raiva transmitida por cães é a vacinação animal em massa, sendo a população canina estimada oficialmente como $10 \%$ da população humana (Tabela 4). Pela estimativa populacional oficial utilizada, alcança-se a meta planejada para bloqueio da transmissão do vírus rábico, porém, pela estimativa populacional adotada nesta pesquisa, essa meta não é atingida em algumas áreas do Estado (Tabela 5). As coberturas vacinais estão aumentando lentamente, sem atingir ainda os índices necessários para o controle da raiva canina.

Após a análise de todos os indicadores e a caracterização das diferentes áreas de risco de transmissão de raiva humana por cães e gatos, observou-se que em Minas Gerais encontra-se graus de risco diferenciados e que as áreas de alto risco coincidem com as maiores áreas de pobreza e analfabetismo do estado. A caracterização das áreas de risco pode ser visualizada na Figura 4.

\section{Discussão}

Há uma subestimativa da população canina calculada pelo índice de $10 \%$ da população humana (MS, 1988), demonstrada pelas coberturas vacinais acima de $100 \%$. Segundo a OPAS
Tabela 2

Taxa de incidência para raiva canina, de acordo com a Diretoria Regional de Saúde. Minas Gerais, Brasil, 1991-1999.

\begin{tabular}{|c|c|c|c|c|}
\hline \multirow{2}{*}{$\begin{array}{l}\text { Diretoria Regional } \\
\text { de Saúde }\end{array}$} & \multicolumn{4}{|c|}{ Incidência de raiva canina $(1: 10.000)$} \\
\hline & $1991-1993$ & 1994-1996 & 1997-1999 & 1991-1999 \\
\hline Alfenas & 0,00 & 0,00 & 0,14 & 0,15 \\
\hline Barbacena & 0,00 & 0,00 & 0,00 & 0,00 \\
\hline Coronel Fabriciano & 0,13 & 0,00 & 0,00 & 0,12 \\
\hline Diamantina & 0,53 & 0,00 & 1,48 & 1,95 \\
\hline Divinópolis & 0,00 & 0,00 & 0,00 & 0,00 \\
\hline Governador Valadares & 0,57 & 0,07 & 3,03 & 3,56 \\
\hline Itabira & 0,31 & 0,00 & 0,00 & 0,31 \\
\hline Ituiutaba & 0,00 & 0,00 & 0,00 & 0,00 \\
\hline Juiz de Fora & 0,00 & 0,00 & 0,17 & 0,18 \\
\hline Leopoldina & 0,00 & 0,00 & 0,00 & 0,00 \\
\hline Manhumirim & 0,00 & 0,00 & 0,59 & 0,58 \\
\hline Metropolitano & 0,00 & 0,00 & 0,00 & 0,00 \\
\hline Montes Claros & 0,47 & 0,04 & 0,00 & 0,50 \\
\hline Passos & 0,00 & 0,00 & 0,00 & 0,00 \\
\hline Patos de Minas & 0,11 & 0,00 & 0,00 & 0,11 \\
\hline Pedra Azul & 2,37 & 0,42 & 6,44 & 3,40 \\
\hline Ponte Nova & 0,00 & 0,00 & 0,00 & 0,00 \\
\hline Pouso Alegre & 0,00 & 0,00 & 0,00 & 0,00 \\
\hline São João Del Rei & 0,00 & 0,00 & 0,00 & 0,00 \\
\hline Sete Lagoas & 0,00 & 0,12 & 0,00 & 0,12 \\
\hline Teófilo Otoni & 1,05 & 3,59 & 1,20 & 5,79 \\
\hline Ubá & 0,00 & 0,00 & 0,00 & 0,00 \\
\hline Uberaba & 0,00 & 0,00 & 0,00 & 0,00 \\
\hline Uberlândia & 0,00 & 0,00 & 0,00 & 0,00 \\
\hline Varginha & 0,00 & 0,00 & 0,00 & 0,00 \\
\hline Total & 0,17 & 0,13 & 0,25 & 0,55 \\
\hline
\end{tabular}

Tabela 3

Distribuição dos casos de raiva humana pelo animal transmissor, por Diretoria Regional de Saúde de residência. Minas Gerais, Brasil, 1991-1999.

\begin{tabular}{|c|c|c|c|c|c|}
\hline $\begin{array}{l}\text { Diretoria Regional } \\
\text { de Saúde }\end{array}$ & Cão & Gato & Morcego & Ignorado & Total \\
\hline Alfenas & 0 & 0 & 1 & 0 & 1 \\
\hline Diamantina & 3 & 0 & 0 & 0 & 3 \\
\hline Governador Valadares & 5 & 0 & 4 & 0 & 9 \\
\hline Montes Claros & 5 & 1 & 1 & 1 & 8 \\
\hline Pedra Azul & 1 & 0 & 0 & 0 & 1 \\
\hline São João Del Rei & 1 & 0 & 0 & 0 & 1 \\
\hline Teófilo Otoni & 6 & 0 & 2 & 1 & 9 \\
\hline Uberlândia & 0 & 0 & 1 & 0 & 1 \\
\hline Total & 21 & 1 & 9 & 2 & 33 \\
\hline
\end{tabular}


Figura 3

Distribuição dos casos notificados de raiva humana transmitidos por cães. Minas Gerais, Brasil, 1991-1999.
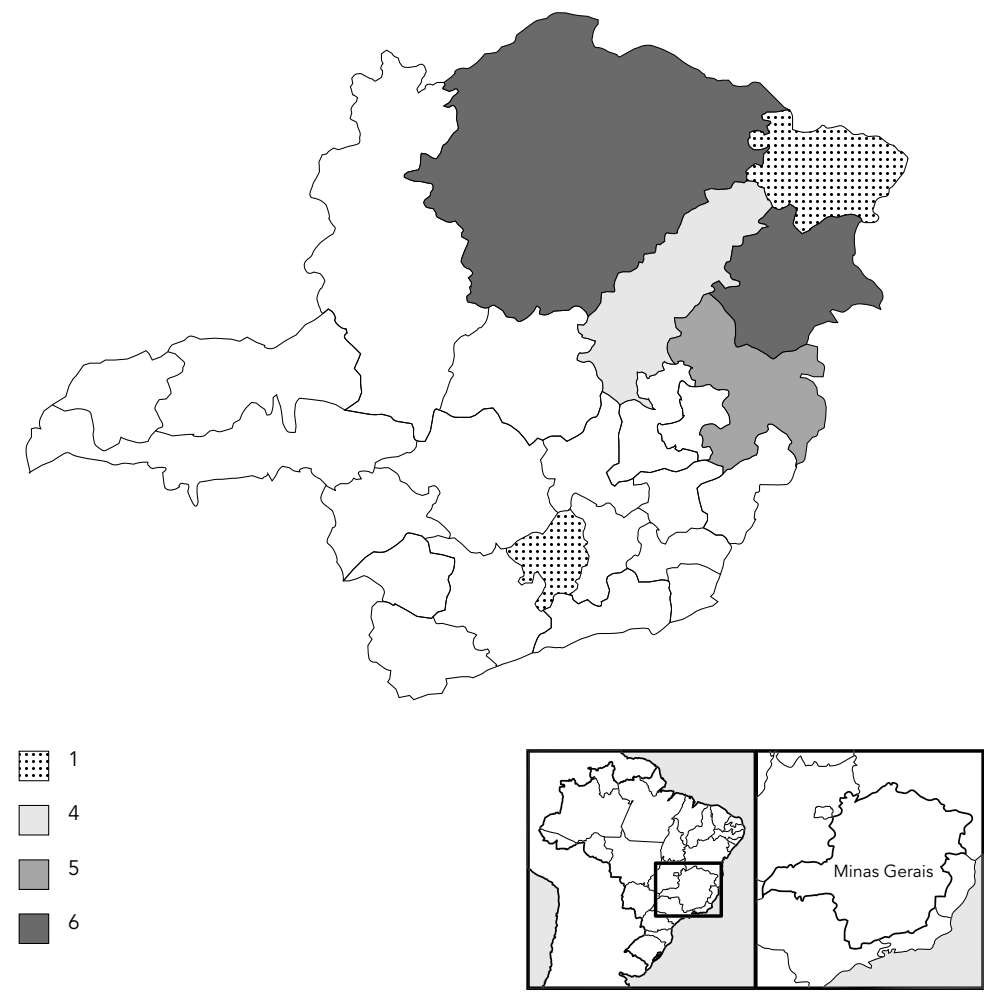

(1992), a relação observada nos países onde esses estudos têm sido feitos, varia entre 1:4 e 1:7. Silva et al. (1982), em Belo Horizonte, Minas Gerais e Reichmann et al. (2000), no Estado de São Paulo, após estudos populacionais, encontraram índice médio de um cão para cada oito habitantes. É necessário levar em consideração as diferenças entre os portes de município e a densidade demográfica em áreas urbanas, suburbanas, periféricas e rurais.

Não existe no estado uma correlação entre concentração da população canina e ocorrência de raiva. Luz (1988) já verificava em 1988, uma tendência na diminuição dos casos de raiva canina da capital e aumento no interior. Segundo a OPAS (1994), essa situação é encontrada em toda a América Latina, onde $89 \%$ dos casos estão ocorrendo em municípios com menos de 50 mil habitantes.

Há probabilidade da ocorrência de casos importados em áreas onde a raiva canina está controlada: Minas Gerais é um elo de integra- ção entre interior-litoral e norte-sul do país, possuindo uma das maiores malhas rodoviárias do Brasil. A raiva canina e felina está diagnosticada laboratorialmente em todos os estados limítrofes, exceto o Rio de Janeiro (Araújo, 2000). Há deficiência no controle zoosanitário nas áreas de divisas estaduais, ignorando a Portaria no 126/76, artigo 27, do Ministério da Agricultura (MAA, 1991). Durante os deslocamentos pelo estado, observou-se o transporte de cães, inclusive em caminhões. Segundo o relato informal de um caminhoneiro, o cão é mantido como animal de companhia e guarda; porém, quando acometidos por alguma enfermidade, são abandonados em postos de gasolina ou restaurantes de beira de estrada. Alguns deles podem estar no período de incubação da doença como o visto no relato de Krebs et al. (1997), que verificou o deslocamento de pessoas transportando animais infectados com a variante do vírus rábico cão/coiote entre diferentes estados dos Estados Unidos. Barreiras naturais são ultrapassadas pela interferência do homem, intensificando a migração canina. O cão vence distâncias pelo acompanhamento do movimento migratório humano que, segundo Armijo-Rojas (1976), levam ao incremento da população canina nas aglomerações marginais urbanas. Esse fato foi constatado no $\mathrm{Mu}$ nicípio de Governador Valadares: estão nessas áreas a maioria dos casos de raiva canina. Barreiras artificiais foram criadas: as estradas de rodagem atuam como um bloqueio na migração de cães errantes. Nota-se um aumento de cães atropelados em rodovias no sentido norte/nordeste de Minas Gerais. O cão raivoso é um animal susceptível ao atropelamento devido à característica neurológica da doença. Apesar dos animais atropelados poderem atuar como sentinelas, não há uma rotina de coleta de material para diagnóstico laboratorial em nenhuma parte do estado.

Nota-se um aumento no envio de amostras em 11 das 25 DRS, sendo que em cinco delas houve ocorrência de diagnóstico positivo para raiva canina. A média anual da população canina enviada para diagnóstico laboratorial é de 0,01\%, pelo índice extra-oficial. Schneider (1990) recomenda a análise de amostras de $0,2 \%$ da população canina.

A situação da raiva no estado coincide com a situação de desenvolvimento regional: áreas mais desenvolvidas, menor a possibilidade de transmissão para o homem; regiões menos desenvolvidas e mais carentes, ocorrência de casos humanos. Esse fato foi verificado em outras partes do mundo por Fekadu (1991). A área com prevalência positiva coincide, entre ou- 
Tabela 4

Estimativa da população canina através da população humana, pelos índices oficiais e extra-oficiais. Minas Gerais, Brasil, 1999.

\begin{tabular}{|c|c|c|c|}
\hline \multirow{2}{*}{$\begin{array}{l}\text { Diretoria Regional } \\
\text { de Saúde }\end{array}$} & \multirow{2}{*}{$\begin{array}{l}\text { População } \\
\text { residente }\end{array}$} & \multicolumn{2}{|c|}{ População canina } \\
\hline & & Oficial & Extra-oficial \\
\hline Alfenas & 413.577 & 41.358 & 70.308 \\
\hline Barbacena & 459.527 & 45.953 & 78.120 \\
\hline Coronel Fabriciano & 508.517 & 50.852 & 86.448 \\
\hline Diamantina & 438.466 & 43.847 & 74.539 \\
\hline Divinópolis & 1.015 .805 & 101.581 & 172.687 \\
\hline Governador Valadares & 791.270 & 79.127 & 134.516 \\
\hline Itabira & 393.746 & 39.375 & 66.937 \\
\hline Ituiutaba & 172.718 & 17.272 & 29.362 \\
\hline Juiz de Fora & 686.443 & 68.644 & 116.695 \\
\hline Leopoldina & 216.940 & 21.694 & 36.880 \\
\hline Manhumirim & 396.801 & 39.680 & 67.456 \\
\hline Metropolitano & 4.229 .410 & 422.941 & 592.117 \\
\hline Montes Claros & 1.401 .529 & 140.153 & 238.260 \\
\hline Passos & 362.180 & 36.218 & 61.571 \\
\hline Patos de Minas & 562.792 & 56.279 & 95.675 \\
\hline Pedra Azul & 274.123 & 27.412 & 46.601 \\
\hline Ponte Nova & 322.377 & 32.238 & 54.804 \\
\hline Pouso Alegre & 784.246 & 78.425 & 133.322 \\
\hline São João Del Rei & 237.741 & 23.774 & 40.416 \\
\hline Sete Lagoas & 545.934 & 54.593 & 92.809 \\
\hline Teófilo Otoni & 486.586 & 48.659 & 82.720 \\
\hline Ubá & 400.832 & 40.083 & 68.141 \\
\hline Uberaba & 586.330 & 58.633 & 99.676 \\
\hline Uberlândia & 834.739 & 83.474 & 141.906 \\
\hline Varginha & 773.326 & 77.333 & 131.465 \\
\hline Total & 17.295 .955 & 1.729 .596 & 2.813 .430 \\
\hline
\end{tabular}

Tabela 5

Cobertura vacinal pela estimativa populacional canina oficial e extra-oficial. Minas Gerais, Brasil, 1997-1999.

\begin{tabular}{|c|c|c|}
\hline \multirow{2}{*}{$\begin{array}{l}\text { Diretoria Regional } \\
\text { de Saúde }\end{array}$} & \multicolumn{2}{|c|}{ Cobertura vacinal (\%) } \\
\hline & Oficial & Extra-oficial \\
\hline Alfenas & 106,70 & 62,77 \\
\hline Barbacena & 129,62 & 75,25 \\
\hline Coronel Fabriciano & 71,55 & 42,09 \\
\hline Diamantina & 102,71 & 60,42 \\
\hline Divinópolis & 111,44 & 65,55 \\
\hline Governador Valadares & 118,50 & 69,71 \\
\hline Itabira & 129,44 & 76,14 \\
\hline Ituiutaba & 125,32 & 73,72 \\
\hline Juiz de Fora & 100,53 & 59,14 \\
\hline Leopoldina & 83,31 & 49,01 \\
\hline Manhumirim & 99,37 & 58,46 \\
\hline Metropolitano & 107,85 & 76,50 \\
\hline Montes Claros & 109,26 & 64,27 \\
\hline Passos & 95,92 & 56,43 \\
\hline Patos de Minas & 95,70 & 56,29 \\
\hline Pedra Azul & 81,62 & 48,01 \\
\hline Ponte Nova & 104,02 & 61,19 \\
\hline Pouso Alegre & 106,64 & 62,73 \\
\hline São João Del Rei & 123,11 & 72,42 \\
\hline Sete Lagoas & 129,80 & 76,35 \\
\hline Teófilo Otoni & 114,69 & 67,61 \\
\hline Ubá & 87,87 & 51,69 \\
\hline Uberaba & 111,00 & 65,29 \\
\hline Uberlândia & 108,89 & 64,05 \\
\hline Varginha & 126,12 & 74,19 \\
\hline Total & 108,31 & 63,71 \\
\hline
\end{tabular}

tros aspectos relacionados com o desenvolvimento social, com a área de maior incidência de índice de analfabetismo do Estado (DATASUS, 2001), e de maior precariedade de acessos. Os casos positivos das outras áreas do Estado ocorreram de forma focal, não indicando um risco iminente da instalação de forma endêmica. O bloqueio realizado no momento do diagnóstico do caso nessas áreas, mostrou-se suficiente para a estagnação do processo de instalação de um novo ciclo, estando o resultado de acordo com o esperado após a aplicação das recomendações da OPAS (PAHO, 1999).

A dominância da espécie canina na transmissão da raiva humana também foi observada por Ribeiro Netto \& Machado (1970), Moreira et al. (1976), Acha \& Szyfres (1986), OPAS (1992) e Araújo (2000).

A vacinação em massa apresenta uma cobertura global inferior à preconizada pela Organização Mundial da Saúde (OMS), quando avaliada pela estimativa de população canina extra-oficial. As DRS com cobertura mais baixa encontram-se limítrofes das áreas endêmicas para raiva canina. As coberturas vêm aumentando, mas ainda não atingiram um nível suficiente para impedir a disseminação do vírus nessas áreas, tornando-se necessário uma vigilância mais efetiva.

Não é realizado um controle na qualidade da vacina. Houve casos de raiva em cães vacinados com três doses, nas DRS Diamantina e Governador Valadares. Não há uma preocupação no manuseio e no armazenamento da vacina de uso animal, comprometendo a qualidade do imunobiológico.

Em relação ao tratamento humano, a indicação não observa a situação epidemiológica local, acarretando um excesso de prescrições em locais onde a raiva canina encontra-se controlada.

Assim, verifica-se que Minas Gerais apresenta todos os graus de risco para raiva huma- 
Figura 4

Caracterização das áreas de risco para raiva humana. Minas Gerais,

Brasil, 1991-1999.

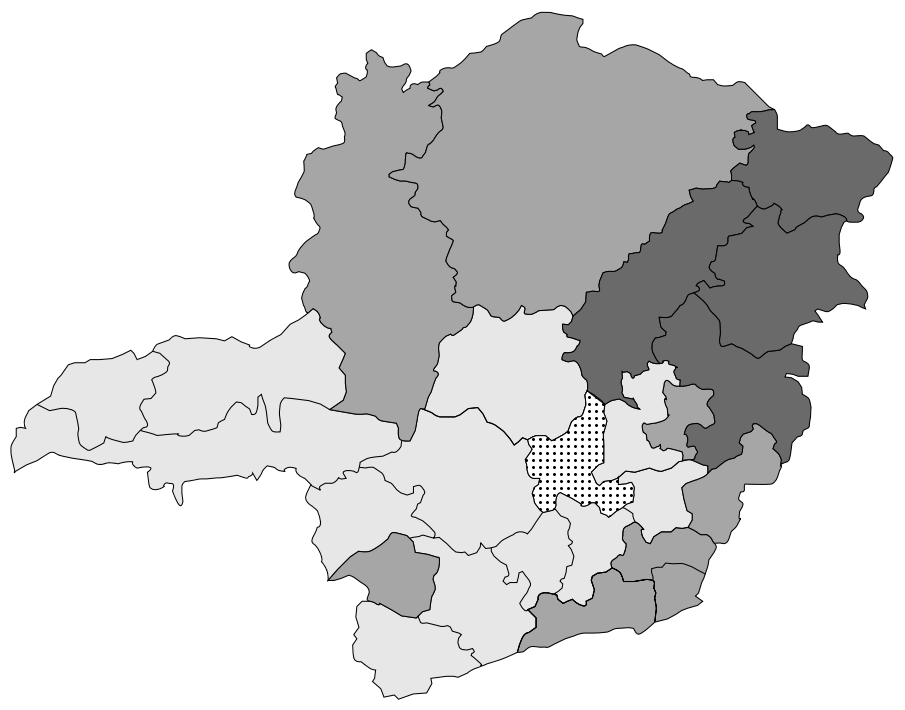

risco nulo

baixo risco

médio risco

alto risco na transmitida por cães, desde o nulo até o mais alto risco. Essa verificação também pode ser observada em outras partes do mundo, conforme assinala Belotto (2000). Em Minas Gerais, há ocorrência de raiva humana nas áreas caracterizadas com risco alto.

O modelo adotado até então para o Programa de Profilaxia da Raiva Humana em Minas Gerais não é baseado na situação epidemiológica diferenciada. O conhecimento das áreas de risco permite a utilização de condutas adaptadas à situação de cada área, estabelecendo prioridades locais e possibilitando uma racionalização dos recursos financeiros que poderiam ser voltados para a implementação da vigilância epidemiológica. Com isso, obtém-se melhor desempenho das ações de impacto, além de diminuir o risco de aparecimento de eventos adversos pela vacina anti-rábica humana em áreas onde a raiva canina encontrase controlada.

\section{Conclusões}

Com base nos resultados, pode-se concluir que: - Existem áreas de risco diferenciadas para raiva humana em Minas Gerais.

- As áreas de maior risco coincidem com as regiões de menor desenvolvimento socioeconômico do estado.

- A cobertura vacinal com base na proporção de um cão para dez habitantes está superestimada, não alcançando o nível real de $75 \%$ da população canina, necessário para controlar e erradicar a raiva urbana.

- Há necessidade de elaboração de um programa de erradicação da raiva canina em Minas Gerais, utilizando-se de estratégias diferenciadas de acordo com as áreas de risco. 


\section{Referências}

ACHA, P. N. \& SZYFRES, B., 1986. Zoonosis y Enfermedades Transmisibles Comunes al Hombre y a los Animales. Publicación Científica 503. 2a Ed. Washington, DC: Organización Panamericana de la Salud.

ARAUJO, F. A., 2000. A situação da raiva no Brasil. In: Seminário Internacional da Raiva, Resumos, p. 22, São Paulo: Instituto Pasteur.

ARMIJO-ROJAS, R., 1976. Epidemiología. Buenos Aires: Intermédica.

BELOTTO, A. J., 2000. Raiva canina - Vigilância epidemiológica e caracterização de áreas de risco. In: Seminário Internacional da Raiva, Resumos, pp. 36-37, São Paulo: Instituto Pasteur.

DATASUS (Departamento de Informática do SUS), 2000. Informações em Saúde. 20 Novembro 2000 $<$ http://www.datasus.gov.br>.

DATASUS (Departamento de Informática do SUS), 2001. Informações em Saúde. 28 Janeiro 2001 $<$ http://www.datasus.gov.br>.

FEKADU, M., 1991. Canine rabies. In: The Natural History of Rabies (G. M. Baer, ed.), pp. 367-378, 2nd Ed., Boca Raton: CRC Press.

IBGE (Fundação Instituto Brasileiro de Geografia e Estatística), 2001. População. 29 Janeiro 2001 $<$ http://www.ibge.gov.br>.

KREBS, J. W.; SMITH, J. S.; RUPPRECHT, C. E. \& CHILD, J. E., 1997. Rabies surveillance in the United States during 1996. Journal of the American Veterinary Medical Association, 211:1525-1539.

LUZ, C. R., 1988. Estudo Cronológico sobre a Raiva em Minas Gerais no Período de 1976 a 1986. Dissertação de Mestrado, Belo Horizonte: Escola de Veterinária, Universidade Federal de Minas Gerais.

MAA (Ministério da Agricultura e do Abastecimento), 1991. Portaria no 126/76. Brasília: MAA.

MS (Ministério da Saúde), 1988. Procedimentos para o Controle de Populações Animais Urbanas. Série Cadernos 3. Brasília: Centro de Documentação, MS.

MOREIRA, E. C.; GONTIJO, M. T.; CASTRO, A.; REIS, R.; VIANA, F. C. \& MOREIRA, W. L., 1976. Aspectos epidemiológicos del tratamiento anti-rábico humano en Belo Horizonte, Minas Gerais, Brasil. Boletín de la Oficina Sanitaria Panamericana, 80:38-44.
OPAS (Organización Panamericana de la Salud), 1992. Plan de Acción para la Consolidación de la Fase de Ataque Final para la Eliminación de la Rabia en las Américas Hasta el Año 2000. Documento de Trabajo. Washington, DC: OPAS/Organización Mundial de la Salud.

OPAS (Organización Panamericana de la Salud), 1994. Reunión de Consulta de Espertos sobre las Bases Técnicas para el Reconocimiento de Areas Libres de Rabia y Requisitos de Cuarentena de Animal. Santo Domingo: OPAS/Organización Mundial de la Salud.

PAHO (Pan American Health Organization), 1999. PAHO/WHO Expert Consultation on Canine Rabies - Free Municipalities. Havana: PAHO/World Health Organization.

REICHMANN, M. L. A. B.; SANDOVAL, M. R. C. \& TAKAOKA, N. Y., 2000. Estratégias de Controle da Raiva Canina - Experiência do Estado de São Paulo. In: Seminário Internacional da Raiva, $R e$ sumos, pp. 40-41, São Paulo: Instituto Pasteur.

RIBEIRO NETTO, A. \& MACHADO, C. G., 1970. Alguns aspectos epidemiológicos da exposição humana ao risco da infecção pelo vírus da raiva na cidade de São Paulo, Brasil. Revista do Instituto de Medicina Tropical de São Paulo, 12:16-30.

SCHNEIDER, M. C., 1990. Estudo de Avaliação sobre Área de Risco de Raiva no Brasil. Dissertação de Mestrado, Rio de Janeiro: Fundação Oswaldo Cruz.

SECRETARIA MUNICIPAL DE SAÚDE DE BELO HORIZONTE, 2000. Censo Canino 2000. Belo Horizonte: Serviço de Controle de Zoonoses, Secretaria Municipal de Saúde de Belo Horizonte.

SES-MG (Secretaria de Estado da Saúde de Minas Gerais), 2000. Consolidados de Vacinação Anti-rábica Animal no Estado de Minas Gerais. Belo Horizonte: Coordenadoria de Zoonoses, SES-MG.

SILVA, J. A.; MOREIRA, E. C.; VIANA, F. C. \& REIS, J. C., 1982. Características da população canina e felina de Belo Horizonte, Minas Gerais - Brasil. Arquivos da Escola de Veterinária, 34:117-126.

TRIVIÑOS, A. N. S., 1987. Introdução à Pesquisa em Ciências Sociais: A Pesquisa Qualitativa em Educação. São Paulo: Ática.

Recebido em 3 de julho de 2001

Versão final reapresentada em 1 de abril de 2002

Aprovado em 4 de julho de 2002 